\title{
MUSINGS
}

\section{Circulating tumor cells versus tumor-derived cell- free DNA: rivals or partners in cancer care in the era of single-cell analysis?}

\author{
Evelyn Kidess ${ }^{1,2}$ and Stefanie S Jeffrey ${ }^{* 1}$
}

Cancer presents a problem of complexity. Although our understanding of tumor biology has increased exponentially over recent decades, and we have many available technologies to characterize tumors, whether and when an individual cancer will metastasize remains unknown. Moreover, if metastases do occur, their molecular features may not match those of the primary tumor or other metastases. Ideally, to guide treatment decisions, all micro- and macrometastases would be identified, biopsied and molecularly analyzed, but this is not currently possible, practical or safe. Thus, investigations are now focusing on blood-based assays that detect and characterize circulating tumor cells or circulating tumor DNA (a component of cell-free DNA). These minimally invasive, real-time 'liquid biopsies' can be performed at multiple intervals to monitor disease and tailor cancer therapy. It is as yet unknown whether these represent competing technologies or if they should be used together.

Tumors are heterogeneous and tend to change over time. As a result of biomechanical and chemical cues, subsets of tumor cells die and others undergo reprogramming, allowing them to survive under adverse conditions, such as cell crowding, low tissue oxygenation, poor nutrient supply and influences from immune modulation. In metastasizing tumors, some cells develop the capacity to penetrate blood vessels, survive the shear forces of the bloodstream, and travel to the bone marrow and distant sites, where a subset is capable of lodging, growing and triggering angiogenic switches in the relatively hostile foreign environment of other organs. For cells that have metastasized beyond their organ of origin, some remain dormant, resistant to adjuvant therapies, whereas others grow and seed new tumor foci that may not respond to cytotoxic drugs or targeted therapies.

\footnotetext{
*Correspondence: ssj@stanford.edu

'Department of Surgery, Stanford University School of Medicine, Stanford, CA 94305, USA

Full list of author information is available at the end of the article
}

For treatment planning, it is impossible to know definitely if surgical excision will cure a given cancer (as it would for a non-metastasizing cancer), or whether tumor cells have already been shed by the primary tumor into the blood and/or lymphatic systems and systemic therapy is required. Once a decision is made to administer systemic therapy, drug selection is generally based on the results of large-scale clinical trials. However, drugs proven effective for an overall population in clinical trials may not work on an individual basis if they do not target the specific biology of a patient's tumor or if the patient metabolically renders the drugs less effective - a basis for pharmacogenomic investigations. On a more granular level, intratumoral drug delivery can vary due to regional differences in blood flow and oxygen levels and/or individual cells can show different sensitivities, so that drugs may only ablate specific subpopulations of tumor cells, allowing other cells to continue to grow, spread and further evolve. It is thus appealing to search for minimally invasive methods to monitor and guide clinical care and drug selection at multiple time points during the course of disease. Technologies that assay blood for potential biomarkers, such as glycosylated proteins, circulating tumor cells (CTCs) and tumor-derived cell-free DNA (cfDNA) are being developed and investigated [1].

\section{Single-cell analysis of circulating tumor cells}

Single-cell analysis enables the identification and characterization of diverse cell populations within tumors and metastases, and among disseminated cancer cells in the blood and bone marrow, dissecting heterogeneity to elucidate biology. Studies in different epithelial cancer types have demonstrated that the number of CTCs detected is related to prognosis $[1,2]$. Beyond enumeration, CTC characterization is key to revealing metastatic biology and guiding therapy. Single-cell applications have been particularly important for analyzing the genomic, transcriptomic, proteomic, and metabolomic profiles of individual CTCs, the signatures of which may be masked by other blood cells present in far greater numbers that are often captured together with CTCs. Transcriptional 
profiling of single CTCs from even the same blood draw reveals heterogeneity [3]. Similarly, array comparative genomic hybridization has demonstrated heterogeneity among multiple CTCs, with some cells showing concordant and others discordant copy number variations when compared to corresponding primary and metastatic tumors. Single-cell mutational analysis of CTCs has also revealed different mutations from those found in the same patient's primary and metastatic tumors; however, targeted ultra-deep sequencing revealed the presence of matching mutations at subclonal levels despite being initially missed, suggesting that only some primary or metastatic tumor cells transition into CTCs that cause tumor progression [4]. In progressive metastatic breast cancer patients with hormone receptor-positive tumors and large numbers of CTCs, only CTC subsets that expressed the surface proteins EPCAM, CD44, CD47 and MET were capable of forming metastases when injected into the bone marrow of xenograft models, providing further evidence that only some CTC populations initiate metastases [5]. It remains to be determined which specific CTCs are responsible for metastatic seeding in different cancer types, particularly in the more common cases where CTC numbers are smaller. It is also important to study label-free CTC-capture technologies that do not rely on particular surface markers such as EPCAM to capture CTCs, because label-free capture may isolate different metastasis-initiating CTC populations. Given the heterogeneous nature of pooled CTCs, techniques that propagate single CTCs in culture (preferably threedimensional culture) would be a major development to advance in vitro and in vivo personalized drug testing.

\section{Assaying tumor-derived cell-free DNA}

Given the many methods and technical challenges involved in isolating and characterizing CTCs, and in light of current advances in next-generation sequencing, clues to tumor activity are being sought through analyses of DNA isolated directly from blood. Although the majority of circulating extracellular DNA is adsorbed to the surface of leukocytes or erythrocytes, a portion can be identified in the plasma, known as plasma DNA or cfDNA. cfDNA can be derived from normal cells, including normal leukocytes that undergo apoptosis, and cancer cells; it is thus detectable in healthy volunteers, patients without cancer, patients with benign tumors, and cancer patients. Circulating tumor DNA (ctDNA) is the portion of circulating DNA specifically derived from cancer cells, and is similarly present both unbound and bound to leukocytes and erythrocytes [6]. A more precise term, then, when referring to unbound ctDNA in the plasma would be tumor-derived cfDNA. It is thought to originate from lytic, apoptotic or necrotic tumor cells, or by active secretion from macrophages that have phagocytized necrotic cells, or from CTCs themselves $[1,7,8]$.

Most tests for the detection of tumor-derived cfDNA target characteristic genetic or epigenetic modifications, such as mutations in tumor-suppressor genes, activated oncogenes, hypermethylation or chromosomal disorders, to guarantee that cancer cells are indeed the source of the detected cfDNA [7]. A recently published prospective, single-center study evaluated three blood-based biomarkers, cfDNA, CA15-3 and CTCs, in women with progressive metastatic breast cancer confirmed by radiologic imaging. The concentration of cfDNA was shown not only to be the most sensitive biomarker, but also to detect disease progression five months earlier than documented by imaging. However, individual somatic alterations had to first be identified to determine which species of cfDNA to monitor [9]. Several groups have shown that mutations leading to therapeutic resistance can be detected in tumor-derived cfDNA up to ten months before detection by imaging, allowing clinicians to change therapy sooner [10-12]. Moreover, targeted therapy itself, in contrast with standard chemotherapy, seems to select for the survival and growth of tumor cells carrying additional mutations associated with resistance to the targeted therapy [10]. Assaying tumor-derived cfDNA is usually dependent on the target being analyzed (for example, $K R A S, B R A F$ and EGFR mutations, panels of multiple gene mutations, and panels of methylation markers); because distinct aberrations may be present in tumor cells at different cancer sites, it is possible that mutations present among all metastatic tumors in a given patient may be revealed within the tumor-derived cfDNA pool. Tumor-derived cfDNA has also been used for early detection of cancer, especially in studies detecting promoter gene hypermethylation of adenomatous polyposis coli $(A P C)$, septin 9 (SEPT9) or estrogen receptor- $\alpha$ (ESR1) [8].

One note of caution is that some specific tumorderived DNA markers may be predominantly present in cell-bound rather than cell-free fractions [6]. Cytotoxic chemotherapy often induces leukocyte and/or erythrocyte apoptosis, which may release cell-bound DNA into plasma and potentially confound tumor-derived cfDNA measurements for those particular markers (an immediate increase in these markers would be due to death of blood cells, rather than reflecting tumor growth).

Given the tiny amounts of tumor-derived cfDNA in circulation, technical issues such as assay sensitivity and the use of targeted versus untargeted (probe-free) methodologies are important for clinical adoption. A critical question to be answered in future clinical trials is whether tumor-derived cfDNA reflects DNA from mixed populations of tumor cells: is it released from tumor cells dying during a given therapy because they are sensitive to 
that therapy, and/or is it derived from tumor cells persisting because they are resistant to the therapy. It also needs to be proven whether changing therapies earlier than timepoints indicated by imaging will indeed affect clinical outcome (at present, discovering metastatic disease earlier does not usually influence survival). Because of its multiple sources, tumor-derived cfDNA has a variable half-life in the circulation, from around 15 minutes to several hours, and the total concentration in a cancer patient's blood has been shown to vary considerably. Therefore, cfDNA alone is not appropriate for evaluation of tumor stage [8].

Other concerns to be addressed in trials will be which mutations or epigenetic alterations should be analyzed for which tumor types, and in which patients. Even if a standard assay panel (a panel of mutational and/or hypermethylation targets) is designed for each tumor type, the assay would still need to be broad, as individual patients with similar cancer types may show different patterns of mutations or epigenetic changes, and some of these aberrations may change over time or be influenced by treatment.

\section{Combined use of CTCs and tumor-derived cell-free DNA}

So how will these technologies fit into the future landscape of cancer monitoring and care? The isolation and characterization of CTCs is more technically challenging than preparing and assaying cfDNA. There are a multitude of CTC-capture technologies in various stages of development and clinical testing. Other than the CellSearch ${ }^{\oplus}$ platform from Veridex (Johnson \& Johnson), which has expanded clearance from the US Food and Drug Administration, most have not yet undergone the rigorous steps involved for qualification as a technique for clinical biomarker detection. These steps include: analytical validation to optimize and standardize CTCcapture conditions and confirm reproducibility in Clinical Laboratory Improvement Amendments (CLIA)-certified laboratories; and then determination of clinical utility in the context of specific patient populations to define true/ false positive and negative rates for defining sensitivity and specificity, positive and negative predictive values, clinical reproducibility, and benefit over other currently used biomarkers [2]. Similarly, tumor-derived cfDNA assays will require analytical validation and proof of clinical utility before they can be approved for use as a clinical biomarker. However, we expect that once definitive panels of targets or probe-free assay methods are developed and successfully tested, clinical biomarker qualification may be more straightforward for cfDNA than for many current CTC technologies, some of which detect CTCs in a given blood draw in only 60 to $80 \%$ of patients with known metastases. Nonetheless, lack of qualification of CTC capture and characterization as a biomarker is not synonymous with lack of future clinical usefulness.

We foresee the following clinical scenario. Future tumor-derived cfDNA target panels will be used to monitor cancer patients during treatment or while they are in remission. However, once there is evidence of therapeutic failure or disease recurrence, single-cell analysis of CTCs may reveal more actionable information to guide drug therapy. We propose that tumor cells persisting in the blood represent those cells resistant to the respective therapy. Thus, molecular analyses of these persisting CTCs may assist in selecting new therapies against targets or pathways shown to be upregulated; use of such therapies may not be apparent by analyses of pooled tumor-derived cfDNA, which more commonly reveals resistance to therapy. Moreover, CTCs lend themselves to future in vitro or in vivo drug sensitivity testing. This might entail testing single CTCs for drug response (not yet technically possible) or growing individual CTCs in culture or in xenografts for future patient-specific and CTC-subpopulation-specific in vitro or in vivo drug testing that would target and eradicate persisting cancer cell populations responsible for recurrence or therapeutic resistance.

Thus, we suggest that future analyses of both tumorderived cfDNA and CTCs will enable earlier diagnosis of primary or recurrent disease, close monitoring of the cancer, and the administration of optimal therapeutic agents.

\section{Abbreviations}

cfDNA, cell-free DNA; CLIA, Clinical Laboratory Improvement Amendments; $\mathrm{CTC}$, circulating tumor cell; ctDNA, circulating tumor DNA.

\section{Competing interests}

SSJ is an inventor of the MagSweeper, a CTC-capture technology. Stanford University has licensed this technology to Illumina, Inc., and receives licensing royalties; SSJ has donated her royalties to support student research programs at The Jackson Laboratory, a nonprofit biomedical research institution. The authors have no relevant affiliations or financial involvement with any organization or entity with a financial interest in or financial conflict with the subject matter or materials discussed in the manuscript.

\section{Acknowledgements}

This article was supported in part by the John and Marva Warnock Cancer Research Fund (SSJ) and fellowship funds from Natalie and Vladimir Ermakoff (EK).

\section{Author details}

'Department of Surgery, Stanford University School of Medicine, Stanford, CA 94305, USA. ²Department of Surgery, Charité University Hospital, 12203 Berlin, Germany.

\section{Published: 13 August 2013}

\section{References}

1. Alix-Panabières $\mathrm{C}$, Schwarzenbach $\mathrm{H}$, Pantel $\mathrm{K}$ : Circulating tumor cells and circulating tumor DNA. Annu Rev Med 2012, 63:199-215.

2. Danila DC, Pantel K, Fleisher M, Scher HI: Circulating tumors cells as biomarkers: progress toward biomarker qualification. Cancer J 2011, 17:438-450. 
3. Powell AA, Talasaz AH, Zhang H, Coram MA, Reddy A, Deng G, Telli ML, Advani RH, Carlson RW, Mollick JA, Sheth S, Kurian AW, Ford JM, Stockdale FE, Quake SR, Pease RF, Mindrinos MN, Bhanot G, Dairkee SH, Davis RW, Jeffrey SS: Single cell profiling of circulating tumor cells: transcriptional heterogeneity and diversity from breast cancer cell lines. PLoS One 2012, 7:e33788.

4. Heitzer E, Auer M, Gasch C, Pichler M, UIz P, Hoffmann EM, Lax S, WaldispuehlGeigl J, Mauermann O, Lackner C, Höfler G, Eisner F, Sill H, Samonigg H, Pantel K, Riethdorf S, Bauernhofer T, Geigl JB, Speicher MR: Complex tumor genomes inferred from single circulating tumor cells by array-CGH and next-generation sequencing. Cancer Res 2013, 73:2965-2975.

5. Baccelli I, Schneeweiss A, Riethdorf S, Stenzinger A, Schillert A, Vogel V, Klein C, Saini M, Bäuerle T, Wallwiener M, Holland-Letz T, Höfner T, Sprick M, Scharpff M, Marmé F, Sinn HP, Pantel K, Weichert W, Trumpp A: Identification of a population of blood circulating tumor cells from breast cancer patients that initiates metastasis in a xenograft assay. Nat Biotechnol 2013, 31:539-544.

6. Skvortsova TE, Rykova EY, Tamkovich SN, Bryzgunova OE, Starikov AV, Kuznetsova NP, Vlassov VV, Laktionov PP: Cell-free and cell-bound circulating DNA in breast tumours: DNA quantification and analysis of tumourrelated gene methylation. Br J Cancer 2006, 94:1492-1495

7. Swarup V, Rajeswari MR: Circulating (cell-free) nucleic acids--a promising, non-invasive tool for early detection of several human diseases. FEBS Lett 2007, 581:795-799.

8. Schwarzenbach $\mathrm{H}$, Hoon DS, Pantel K: Cell-free nucleic acids as biomarkers in cancer patients. Nat Rev Cancer 2011, 11:426-437.
9. Dawson SJ, Tsui DW, Murtaza M, Biggs H, Rueda OM, Chin SF, Dunning MJ, Gale D, Forshew T, Mahler-Araujo B, Rajan S, Humphray S, Becq J, Halsall D, Wallis M, Bentley D, Caldas C, Rosenfeld N: Analysis of circulating tumor DNA to monitor metastatic breast cancer. N Engl J Med 2013, 368:1199-1209.

10. Misale S, Yaeger R, Hobor S, Scala E, Janakiraman M, Liska D, Valtorta E, Schiavo R, Buscarino M, Siravegna G, Bencardino K, Cercek A, Chen CT, Veronese S, Zanon C, Sartore-Bianchi A, Gambacorta M, Gallicchio M, Vakiani E, Boscaro V, Medico E, Weiser M, Siens S, Di Nicolantonio F, Solit D, Bardelli A: Emergence of KRAS mutations and acquired resistance to anti-EGFR therapy in colorectal cancer. Nature 2012, 486:532-536.

11. Diaz LA Jr, Williams RT, Wu J, Kinde I, Hecht JR, Berlin J, Allen B, Bozic I, Reiter JG, Nowak MA, Kinzler KW, Oliner KS, Vogelstein B: The molecular evolution of acquired resistance to targeted EGFR blockade in colorectal cancers. Nature 2012, 486:537-540.

12. Murtaza M, Dawson SJ, Tsui DW, Gale D, Forshew T, Piskorz AM, Parkinson C, Chin SF, Kingsbury Z, Wong AS, Marass F, Humphray S, Hadfield J, Bentley D, Chin TM, Brenton JD, Caldas C, Rosenfeld N: Non-invasive analysis of acquired resistance to cancer therapy by sequencing of plasma DNA. Nature 2013, 497:108-112.

doi:10.1186/gm474

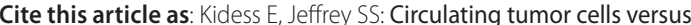
tumor-derived cell-free DNA: rivals or partners in cancer care in the era of single-cell analysis? Genome Medicine 2013, 5:70 